\title{
Enhanced inhibitor tolerance and increased lipid productivity through adaptive laboratory evolution in the oleaginous yeast Metshnikowia pulcherrima
}

Robert H. Hicks, ${ }^{\text {a }}$ Yuxin Sze, ${ }^{\text {b }}$ Christopher J. Chuck, ${ }^{c}$ Daniel A. Henk. ${ }^{b *}$

${ }^{a}$ Centre for Doctoral Training in Sustainable Chemical Technologies, Department of Biology and Biochemistry, University of Bath, Bath, BA2 7AY, United Kingdom

${ }^{\mathrm{b}}$ Department of Biology and Biochemistry, University of Bath, Bath, BA2 7AY, United Kingdom

${ }^{\mathrm{c}}$ Department of Chemical Engineering, University of Bath, Bath, BA2 7AY, United Kingdom

\section{Abstract}

Microbial lipid production from second generation feedstocks presents a sustainable route to future fuels, foods and bulk chemicals. The oleaginous yeast Metshnikowia pulcherrima has previously been investigated as a potential platform organism for lipid production due to its ability to be grown in nonsterile conditions and metabolising a wide range of oligo- and monosaccharide carbon sources within lignocellulosic hydrolysates. However, the generation of inhibitors from depolymerisation causes downstream bioprocessing complications, and despite $M$. pulcherrima's comparative tolerance, their presence is deleterious to both biomass and lipid formation. Using either a single inhibitor (formic acid) or an inhibitor cocktail (formic acid, acetic acid, fufural and HMF), two strategies of adaptive laboratory evolution were performed to improve $M$. pulcherrima's fermentation inhibitor tolerance. Using a sequential batch culturing approach, the resulting strains from both strategies had increased growth rates and reduced lag times under inhibiting conditions versus the progenitor. Interestingly, the lipid production of the inhibitor cocktail evolved strains markedly increased, with one strain producing $41 \%$ lipid by dry weight compared to $22 \%$ of the progenitor. The evolved species was cultured in a non-sterile $2 \mathrm{~L}$ stirred tank bioreactor and accumulated lipid rapidly, yielding $6.1 \mathrm{~g} / \mathrm{L}$ of lipid (35\% cell dry weight) within 48 hours; a lipid productivity of $0.128 \mathrm{~g} \mathrm{L-1} \mathrm{h-1.} \mathrm{Furthermore,} \mathrm{the}$ lipid profile was analogous to palm oil, consisting of $39 \%$ C16:0 and $56 \%$ C18:1 after 48 hours.

\section{Key words:}

Adaptive laboratory evolution, Microbial Oil, Lignocellulose, Fermentation Inhibitors, Bioprocessing, Biodiesel 


\section{Introduction}

Microbial lipids, produced from heterotrophic organisms, are a versatile chemical feedstock that can be used to produce alternatives to fossil derived fuels and chemicals. In comparison to higher plant oils, such as palm oil, microbial oils can be produced on non-arable land, that does not compete with virgin rainforest or food production. While the lipid profile from microalgae is highly variable, generally, oleaginous yeast produce lipid profiles akin to plant oils with elevated levels of oleic and palmitic acid (Sitepu et al. 2014). Recently, we reported on the oleaginous yeast Metschnikowia pulcherrima that can be grown in non-sterile conditions, while having the ability to metabolise a range of oligosaccharide and monosaccharide carbon sources (Whiffin et al. 2016; Long et al. 2017; Fan et al. 2018). Despite this potential, for microbial lipids to financially compete with both fossil and vegetable oils, a low cost lignocellulosic feedstock must be used as the feedstock source.

Processing lignocellulosic feedstocks presents multiple challenges, however, one key issue is the formation of inhibitory by-products resulting from the degradation of monosaccharides produced from the cellulose and hemicellulose feedstocks. These inhibitors halt or inhibit growth and metabolism, slow down productivity and reduce overall product yields (Klinke et al. 2004). Fermentation inhibitors can be classified according to their functional group; carboxylic acid, ketone, phenolic or aldehyde (Taylor et al. 2012). Two of the most abundant are 5-hydroxymethyl furfural (HMF) and 2-furaldehyde (furfural), resulting from the dehydration of hexose or pentose sugars respectively. The most common inhibitory acids produced are formic and acetic acid, with formic acid produced as a derivative of furfural or HMF, and acetic acid produced when acetyl groups are released from hemicellulose (Radecka et al. 2015).

The inhibitory mechanism for these components is complex. HMF and furfural are non-specifically reactive with RNA, DNA and proteins as well as causing membrane damage resulting in disruptions to metabolism and cell viability (Lin et al. 2009). Acetic and formic acid can enter yeast cells via diffusion through the plasma membrane where they dissociate into acetate/formate and a proton once within the cytoplasm. Accumulation of protons leads to cytoplasm acidification causing metabolism impairment by inhibiting glycolytic enzymes and NADH dehydrogenases to increase lag times and reduce growth (Radecka et al. 2015). In addition to the individual effects of each inhibitor, they act synergistically to create negative effects that exceed the sum of each individual effect (Martín et al. 2007; Field et al. 2015).

A rational engineering approach to improve inhibitor tolerance is complex due to the vast network of molecular mechanisms involved. In addition, as genetic toolkits to accomplish such aims are 
unavailable within many non-model organisms, alternative phenotypic improvement strategies, such as adaptive laboratory evolution (ALE), are potentially more promising. ALE is a method whereby microorganisms are continually cultured under a selective pressure in defined conditions for periods of time to allow for the selection of advantageous phenotypes (Dragosits and Mattanovich 2013). Whilst ALE often begins with a natural isolate, it is not uncommon to first perform random mutagenesis through chemical or UV means. Many different evolution strategies have been applied to improve the biotechnological capacity of natural or genetically engineered Saccharomyces cerevisiae strains, for example improving tolerance to ethanol (Stanley et al. 2010) or lignocellulosic fermentation inhibitors (Wright et al. 2011) as well as improving the fermentation of non-preferred sugars such as xylose (Van Maris et al. 2007) and arabinose (Wisselink et al. 2007). There are limited examples of strain improvement through ALE within oleaginous yeast, though Rhodococcus opacus strains which had first undergone genetic engineering followed by mutagenesis selection were further improved by through sequentially batch culturing within increasing concentrations of lignin (Kurosawa et al. 2015).

Oleaginous yeast such as Rhodotorula glutinis, Yarrowia lipolytica and Lipomyces starkeyi tend to have a higher inhibitory tolerance compared to S. cerevisiae (Sitepu et al. 2014; Whiffin et al. 2016). Similarly, Metschnikowia pulcherrima displays a naturally high tolerance to furfural, acetic acid and HMF (Long et al. 2017). M. pulcherrima's industrial potential is further improved by its inherent antimicrobial activity, allowing low cost non-sterile cultures to be performed (Santamauro et al. 2014).

The aim of this current study was to increase the fermentation inhibitor tolerance of $M$. pulcherrima whilst comparing two ALE strategies; either using a fermentation inhibitor cocktail (containing furfural, HMF, formic acid and acetic acid) as a selective pressure, or formic acid in isolation.

\section{Methods.}

\section{Chemicals.}

Unless otherwise stated, chemicals were sourced from Sigma Aldrich and used without further purification.

\section{Strains, strain maintenance and media.}

The M. pulcherrima strain (NCYC2580) used as the progenitor in this study was obtained from the National Collection of Yeast Cultures. Strains were maintained on malt extract agar (MEA) plates, and re-streaked on a fortnightly basis. For the preparation of overnight cultures, a single colony was inoculated into $10 \mathrm{~mL} \mathrm{SMB} \mathrm{pH} 5$ (3\% tryptic soy broth, $2.5 \%$ malt extract), and incubated at $25^{\circ} \mathrm{C}$ with 
$200 \mathrm{rpm}$ agitation. Optical densities throughout were measured at $595_{\mathrm{nm}}$. Media used within ALE study was a nitrogen limited broth (NLB) consisting of: Glucose $40 \mathrm{~g} / \mathrm{L},\left(\mathrm{NH}_{4}\right)_{2} \mathrm{SO}_{2} 2 \mathrm{~g} / \mathrm{L}, \mathrm{KH}_{2} \mathrm{PO}_{4} 7 \mathrm{~g} / \mathrm{L}, \mathrm{MgSO}_{4}$ $7 \mathrm{H}_{2} \mathrm{O} 1.5 \mathrm{~g} / \mathrm{L}, \mathrm{NaHPO}_{2} 2 \mathrm{~g} / \mathrm{L}$ and yeast extract $1 \mathrm{~g} / \mathrm{L}$. This media was autoclaved without glucose or $\mathrm{MgSO}_{4}$, which was added separately after autoclaving individual stock solutions. YNB medium was prepared as follows $-1.78 \mathrm{~g} / \mathrm{L}$ Yeast Nitrogen Base w/o amino acids, $25 \mathrm{~g} / \mathrm{L}$ glucose and variable amounts of $(\mathrm{NH} 4) 2 \mathrm{SO} 2$ per desired concentration.

\section{Evolution experiment.}

Three growth conditions were used for the initial screening experiment: NLB, NLB $+0.6 \mathrm{~g} / \mathrm{L}$ formic acid and NLB + inhibitor cocktail ( $0.7 \mathrm{~g} / \mathrm{L}$ of furfural and acetic acid, and $0.35 \mathrm{~g} / \mathrm{L}$ formic acid and HMF). Triplicate overnight cultures grown in SMB were diluted to an OD 1 with PBS the following morning. Each diluted culture was used to inoculate each of the three screening conditions by adding $500 \mu \mathrm{L}$ into $10 \mathrm{~mL}$ media within a glass culture tube. Cultures were incubated at $25^{\circ} \mathrm{C}$ with $200 \mathrm{rpm}$ agitation with OD measurements were performed at regular intervals through the 72 hour period.

For the ALE experiment, cultures were started as above using the same starting formic acid and inhibitor cocktail concentrations, with five replicate lineages for each condition. OD measurements of each culture were taken at 24 and $48 \mathrm{~h}$ intervals, with cultures transferred after 48 hours by inoculating $100 \mu \mathrm{L}$ of each culture into a fresh $10 \mathrm{~mL}$ culture tube, taking a glycerol stock at each transfer. In the event of no growth in one or more of the culture tubes after 48 hours, successful lineages were expanded to seed new tubes to replace those lost, maintaining a total of five culture tubes per experiment. Inhibitor concentrations were increased when batches were reaching stationary phase after 48 hours to ensure that as much as practically possible exponentially growing cells were being transferred to the next batch. The evolution experiment was concluded after approximately 1000 hours where the final strains were glycerol stocked, and plated onto MEA to provide single colonies for phenotypic analysis.

\section{6 well plate growth phenotypic assays.}

To phenotypically analyse the resulting mutant cell lines, a 96 well plate format was used. To perform these, triplicate overnight cultures for each mutant strain, as well as the progenitor were prepared as stated previously, with each diluted to an $\mathrm{OD} \sim 1$ the following morning. To prepare the 96 well plate, $140 \mu \mathrm{L}$ of media was added to the appropriate well, and to this, $10 \mu \mathrm{L}$ of each diluted culture was added. Media blanks were plated as negative controls. Cultures were incubated within a shaking plate reader with temperature maintained at $25{ }^{\circ} \mathrm{C}$. Optical density was read at 30 minute intervals, for a period of 48 hours unless otherwise stated within figure legends. Max growth rates were calculated 
using RStudio (RStudio 2015), using Ln(OD) values. Media composition and inhibitor concentrations used within the phenotypic screening assays are described within figure legends.

\section{Lipid extraction and profiling.}

Overnight cultures were prepared as previously stated, with $500 \mu \mathrm{L}$ of the diluted overnight culture used to inoculate $10 \mathrm{~mL}$ of NLB or NLB supplemented with an inhibitor cocktail (concentrations used for this assay were: Acetic acid and furfural $0.7 \mathrm{~g} / \mathrm{L}$, formic acid and HMF $0.35 \mathrm{~g} / \mathrm{L}$.

The lipid extraction methodology is based upon that proposed by Bligh and Dyer (Bligh and Dyer 1959), and modified to the following:

Upon completion of culturing, $9 \mathrm{~mL}$ of cells were centrifuged at 13 thousand $\mathrm{rpm}$, resuspended in 1 $\mathrm{mL}$ PBS, and centrifuged again, discarding the supernatant and freezing cell pellets instantly in liquid nitrogen. Following this, frozen cell pellets were freeze dried at $-40^{\circ} \mathrm{C}$ for a minimum of six hours. For the cell disruption, a preweighed amount of cell pellet (ideally within the range of $10-100 \mathrm{mg}$ ) was mixed with $10 \mathrm{~mL} 6 \mathrm{M} \mathrm{HCl}$, and stirred at $80{ }^{\circ} \mathrm{C}$ for one hour. To extract lipids, $10 \mathrm{~mL}$ of chloroform:methanol (1:1) was added, and the mixture was stirred overnight at room temperature. To quantify the lipid weight, the lower chloroform phase was removed by hand with a glass pipette, avoiding the emulsion layer which forms between the chloroform and aqueous phase. The chloroform was then fully evaporated via rotary evaporator at $50{ }^{\circ} \mathrm{C}$, and the remaining lipids were weighed to determine the lipid weight $\%$ of the cells.

For the lipid fatty acid profile, extracted lipid samples were transesterified with methanol and an additional $1 \% \mathrm{H}_{2} \mathrm{SO}_{4}$, heated at $90{ }^{\circ} \mathrm{C}$ under pressure for three hours. The resulting fatty acid methyl esters were extracted with hexane, which was washed with water to remove any residual glycerol or sulfuric acid. GC-MS analysis was carried out using the Agilent 7890A Gas Chromatograph equipped with a CP-Sil capillary column ( $25 \mathrm{~m} \times 0.250 \mathrm{~mm}$ internal diameter) and a He mobile phase (flow rate: $1.2 \mathrm{ml} \mathrm{min}^{-1}$ ), coupled with an Agilent 5975C MSD. Approximately $50 \mathrm{mg}$ of each sample was dissolved in $100 \mathrm{ml}$ hexane and $1 \mu \mathrm{l}$ of each solution was loaded onto the column, pre-heated to $40{ }^{\circ} \mathrm{C}$. This temperature was held for 1 minute and then heated to $250{ }^{\circ} \mathrm{C}$ at a rate of $10{ }^{\circ} \mathrm{C} \mathrm{min}^{-1}$ and then held for 10 minutes. The FAME profile was calculated in reference to known standards.

\section{Bioreactor culturing.}

Overnight cultures were inoculated using a $30 \mathrm{~mL} \mathrm{SMB}$ culture with an OD between 7-10 which was centrifuged and resuspended in $10 \mathrm{~mL}$ PBS. Culturing volume used was $1 \mathrm{~L}$, with $5 \mathrm{~mL}$ polypropylene glycol P2000 (antifoam) added upon inoculation. 80\% oxygenation was maintained at the determined 
concentration through agitation (900 rpm max) and sparging $(\max 3: 1 \mathrm{v} / \mathrm{v}), \mathrm{pH}$ was maintained at 4 through the addition of nitric acid (1M) or sodium hydroxide (1M) and temperature was maintained at $20^{\circ} \mathrm{C}$. These parameters were controlled by the Fermac 320 bioreactor control unit. The cultures were performed to replicate low-cost industrial conditions, and as such, were under non-sterile conditions. Media used was NLB, or NLB supplemented with $0.7 \mathrm{~g} / \mathrm{L}$ of furfural and acetic acid, and $0.35 \mathrm{~g} / \mathrm{L}$ formic acid and HMF.

\section{Results.}

\section{NCYC2580 inhibitor tolerance and adaptive laboratory evolution.}

Concentrations of $0.6 \mathrm{~g} / \mathrm{L}$ formic acid for the single inhibitor strategy and an inhibitor cocktail containing $0.7 \mathrm{~g} / \mathrm{L}$ of furfural and acetic acid $+0.35 \mathrm{~g} / \mathrm{L}$ formic acid and HMF for the multi inhibitor investigation were found to reduce $48 \mathrm{~h}$ growth by approximately $50 \%$ compared to NLB without inhibitors, and therefore chosen as the starting concentrations for the ALE experiments (Figure 1). Inhibitor cocktail cultures displayed an extended lag time compared with the other two conditions, failing to reach an OD greater than 1 until approximately 29 hours. Despite not causing an increased lag time, the formic acid supplemented cultures instead appear exhibit biphasic growth.

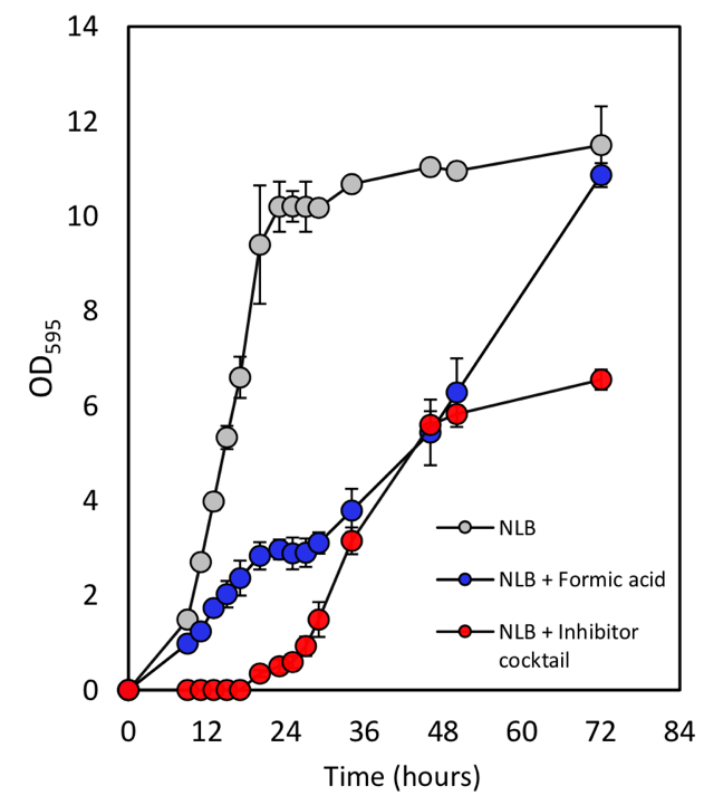

Fig. 1

Growth profiles of NCYC2580 in the presence of inhibitors. Strains were grown in NLB as a control (grey circles), NLB with $0.6 \mathrm{~g} / \mathrm{L}$ formic acid (blue circles) and NLB with an inhibitor cocktail $(0.7 \mathrm{~g} / \mathrm{L}$ furfural and acetic acid, and $0.35 \mathrm{~g} / \mathrm{L}$ formic acid and HMF; red circles). Error bars represent the mean and standard deviation of triplicate culture 
For both ALE experiments, five clonal lineages were started in parallel, and in the event of lineage loss, a successful culture was expanded to maintain a total of five cultures. Cultures were transferred after $48 \mathrm{~h}$, with $100 \mu \mathrm{L}$ of undiluted culture used to inoculate the following batch. Inhibitor concentrations were increased when the 48 hour OD indicated stationary phase was being reached, as determined by the control growth curve in Figure 1. Using this approach, the majority of cells transferred to the new batch would be growing exponentially.

\section{ALE: Formic acid.}

With a starting concentration of $0.6 \mathrm{~g} / \mathrm{L}$ formic acid, OD was consistently high and the concentration across all five lineages was increased to $0.9 \mathrm{~g} / \mathrm{L}$ at batch 4 (Figure 2). Increasing the formic acid concentration to $1 \mathrm{~g} / \mathrm{L}$ at batch 10 resulted in reduced growth, characterised by an increased lag time (shown by the $24 \mathrm{~h}$ sample) in batches 10 and 11, and a decrease in the transfer OD through batches 12 to 14 . All five cultures stabilised from batch 15 onwards, allowing the concentration to be increased further. Though the initial batch at $1.2 \mathrm{~g} / \mathrm{L}$ formic acid saw reduced growth across all lineages, the following four batches recovered, indicating adaptation to this concentration. In total, 22 batches were performed within which a doubling of the initial formic acid concentration of $0.6 \mathrm{~g} / \mathrm{L}$ to $1.2 \mathrm{~g} / \mathrm{L}$ was achieved.

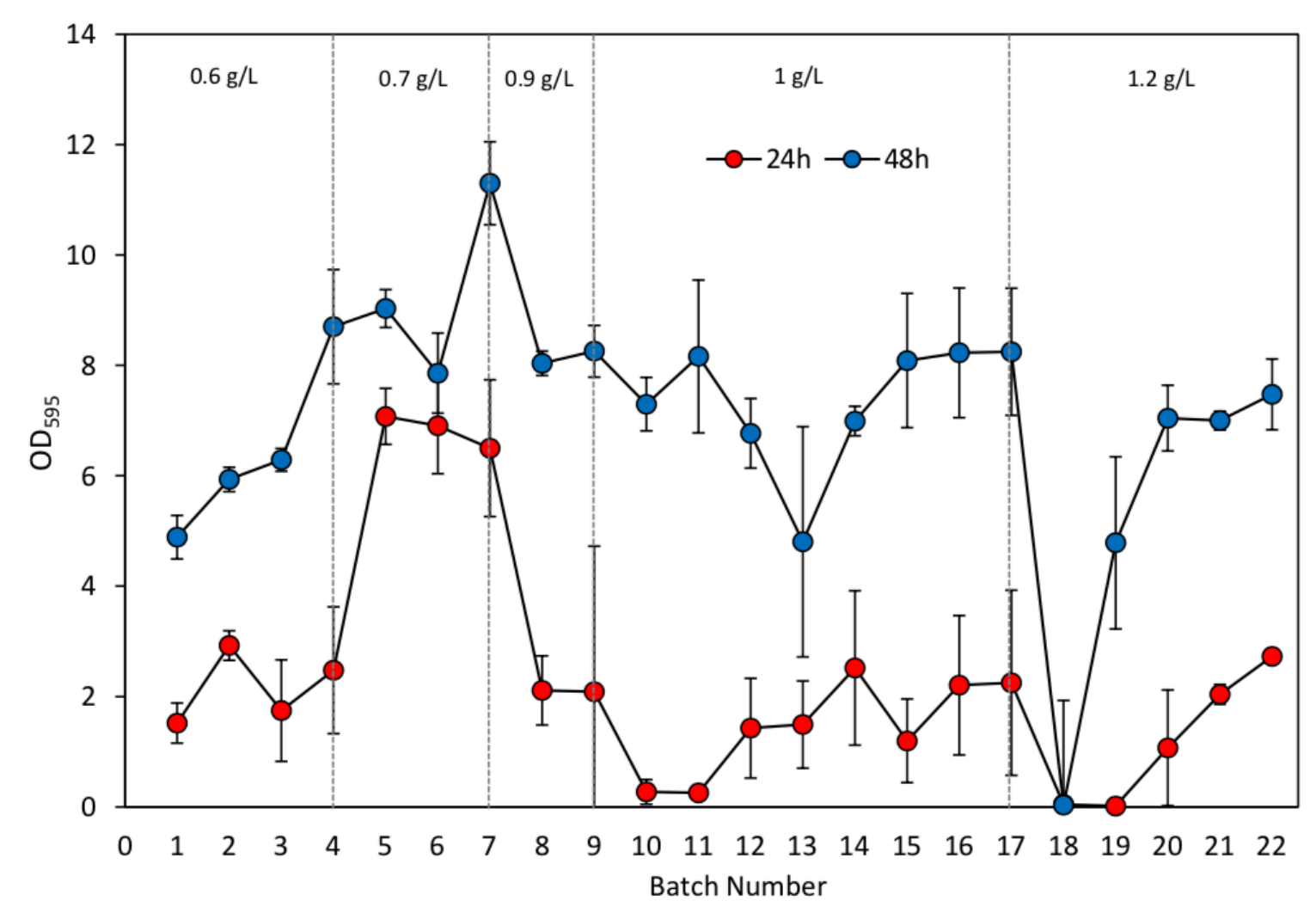


197

198

199

200

201

202

203

204

205

206

207

208

209

210

211

212

213

\section{Fig. 2}

Overview of the NCYC2580 formic acid adaptive laboratory evolution experiment. Five colonies were used to inoculate five individual NLB $+0.6 \mathrm{~g} / \mathrm{L}$ formic acid cultures. Cultures were transferred to fresh media after $48 \mathrm{~h}$ growth; red and blue circles represent the OD values taken at 24 and 48 hours respectively for each batch. All five lineages continued throughout the duration of experiment, therefore error bars represent the mean and standard deviation of five cultures. The concentration of formic acid present in each batch as the experiment progresses is indicated within the plot

\section{ALE: Inhibitor cocktail}

The second ALE strategy applied a cocktail of four inhibitors as the selective pressure, which unlike the formic acid experiment, saw frequent lineage loss within the first 9 batches (Figure 3a). In total, only one of the initial five lineages completed the entire ALE experiment, itself frequently used to expand out to five cultures. The OD data correlates with this batch to batch instability, with large fluctuations in both the 24 and 48 hour OD sample for the first 11 batches (Figure 3b). The trend onwards suggests that adaptation to these conditions; for instance, increases to 24 hour OD measurements indicated that advantageous adaptations were occurring to favour reduced lag time.

These results were in correlation with that presented in Figure $3 a$.

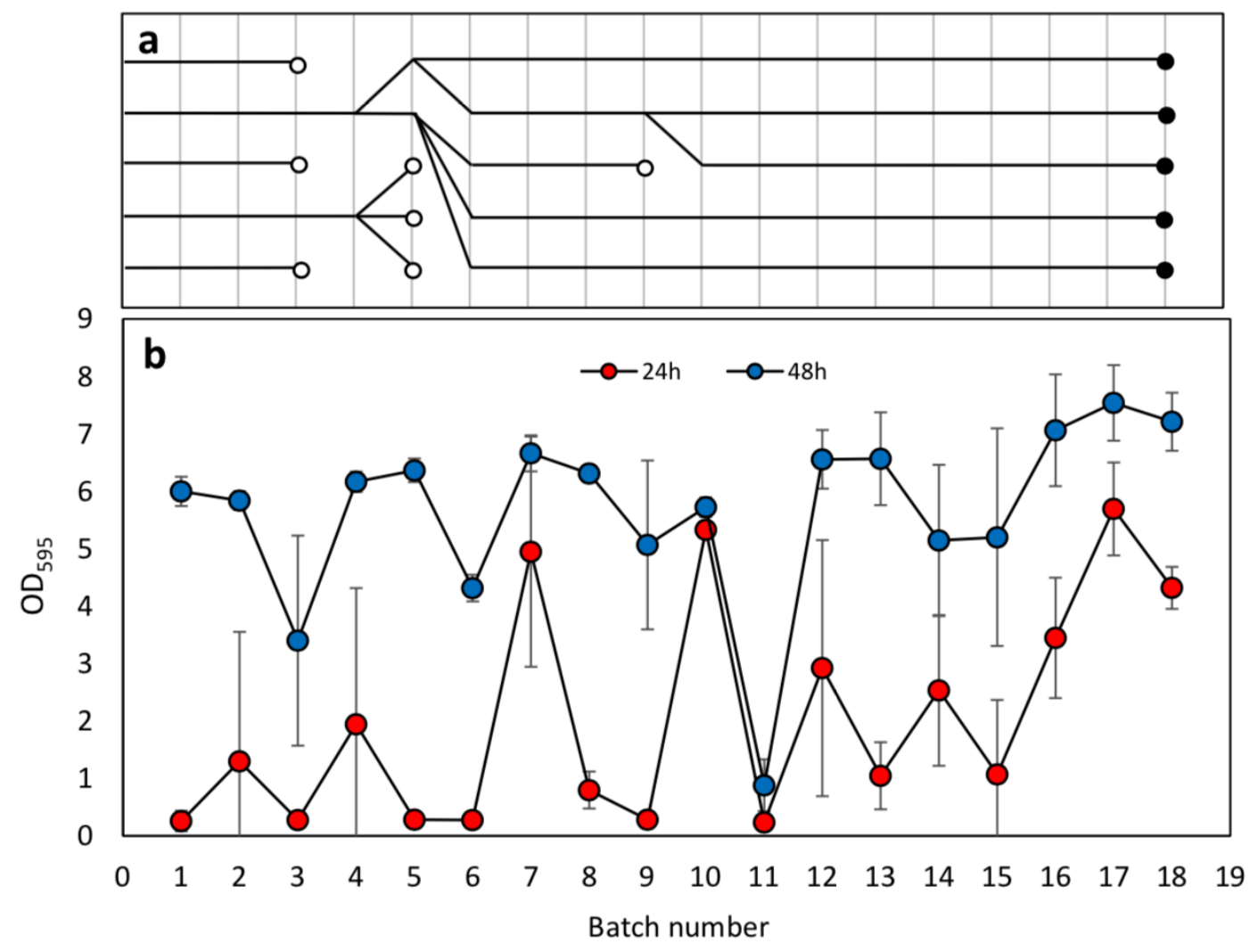


Overview of the NCYC2580 inhibitor cocktail adaptive laboratory evolution experiment. Five individual colonies were used to inoculate five NLB + inhibitor cocktail $(0.7 \mathrm{~g} / \mathrm{L}$ of furfural and acetic acid, and $0.35 \mathrm{~g} / \mathrm{L}$ formic acid and HMF) cultures. a - ALE lineage tree showing successful batches replacing failed batches (clear circles) to maintain five cultures. $b$ - Cultures were transferred to fresh media after $48 \mathrm{~h}$; red and blue circles represent the OD values taken at 24 and 48 hours respectively for each batch. Error bars represent the mean and standard deviation of five cultures

\section{Phenotypic Analysis of Evolved Strains}

Upon completion of both ALE experiments, a streak plate was made from each final batch culture onto MEA agar, and a single colony from each plate was isolated onto a new MEA plate. Four out of the five lineages from each evolution strategy were taken forward for phenotypic analysis and compared to the progenitor strain. They were named as follows:

Formic acid evolved strains: F1, F2, F3, F4.

Inhibitor cocktail evolved strains: $4 \times 1,4 \times 2,4 \times 3,4 \times 4$.

Maximum growth rates in both rich (SMB) and defined (YNB + glucose) media determined that the evolved strains performed comparably to the progenitor when assayed in media different from the evolutionary experiments, suggesting that no discernible evolutionary trade off within these two medias had occurred (Figure 4). Strains were assayed in NLB supplemented with an inhibitor cocktail of $1 \mathrm{~g} / \mathrm{L}$ acetic acid and furfural, and $0.5 \mathrm{~g} / \mathrm{L}$ formic acid and HMF. In this medium, differences in the growth rate were observed, with strains evolved to the inhibitor cocktail displaying maximum growth rates double that shown by the progenitor strain (Figure 5a). The strains evolved within formic acid supplemented media also showed increased growth rates versus the progenitor, though to a lesser extent. Only the formic acid evolved strains showed increased growth rates when assayed in NLB + $0.85 \mathrm{~g} / \mathrm{L}$ formic acid (Figure 5c) meaning that despite both evolution strategies yielding strains with increased growth rates when assayed with an inhibitor cocktail, here, only the formic acid evolved strains showed an increased growth rate. A decrease in the lag time across all evolved strains versus the progenitor was observed in both conditions (Figure $5 b$ and $5 d$ ). This was most notable when grown in NLB + inhibitor cocktail where the lag time reduced by over half, with growth occurring as early as 13 hours post inoculation versus around 40 hours for the progenitor. 

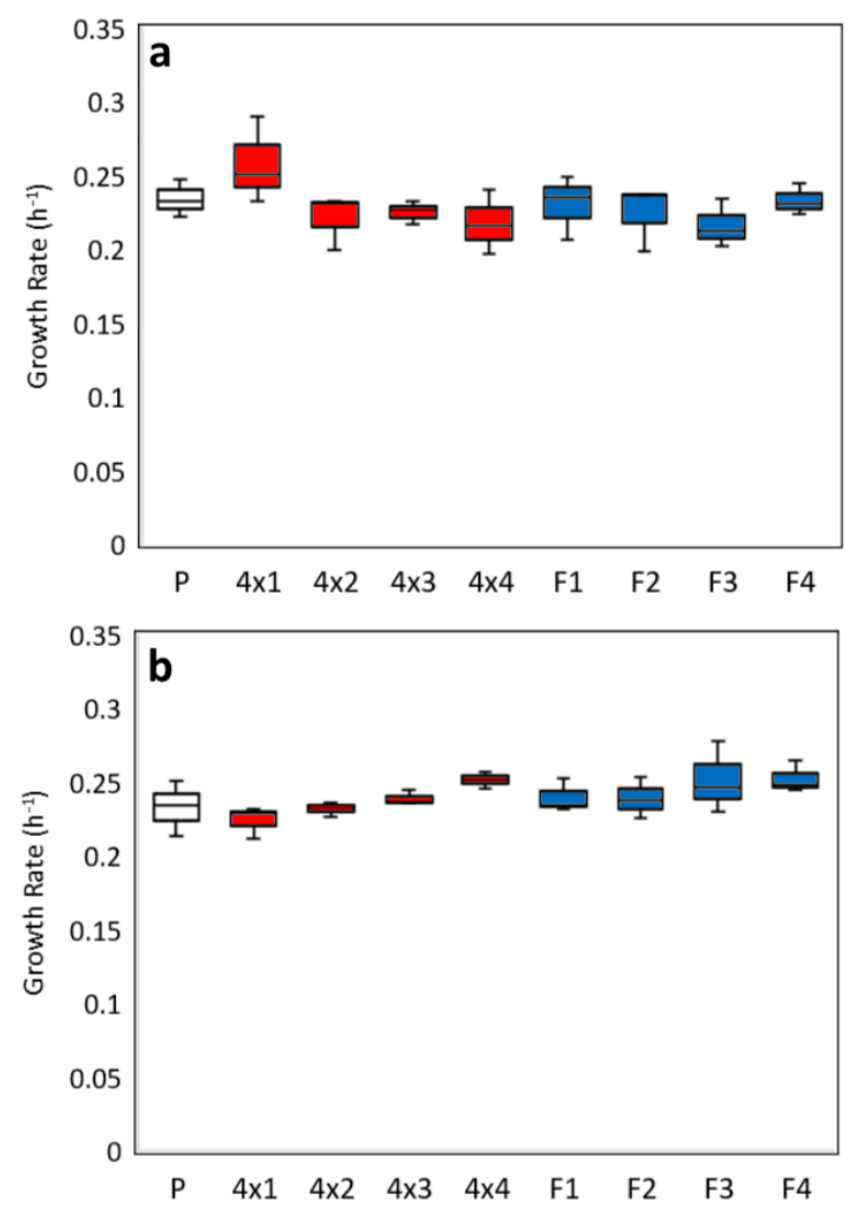

$246 \quad$ Fig. 4

247 Maximum growth rates of eight mutant NYC2580 strains and the progenitor in (a) SMB and (b) YNB + glucose. Each strain was assayed in triplicate with growth performed within a 96 well plate. Max growth rates were calculated using R Studio 

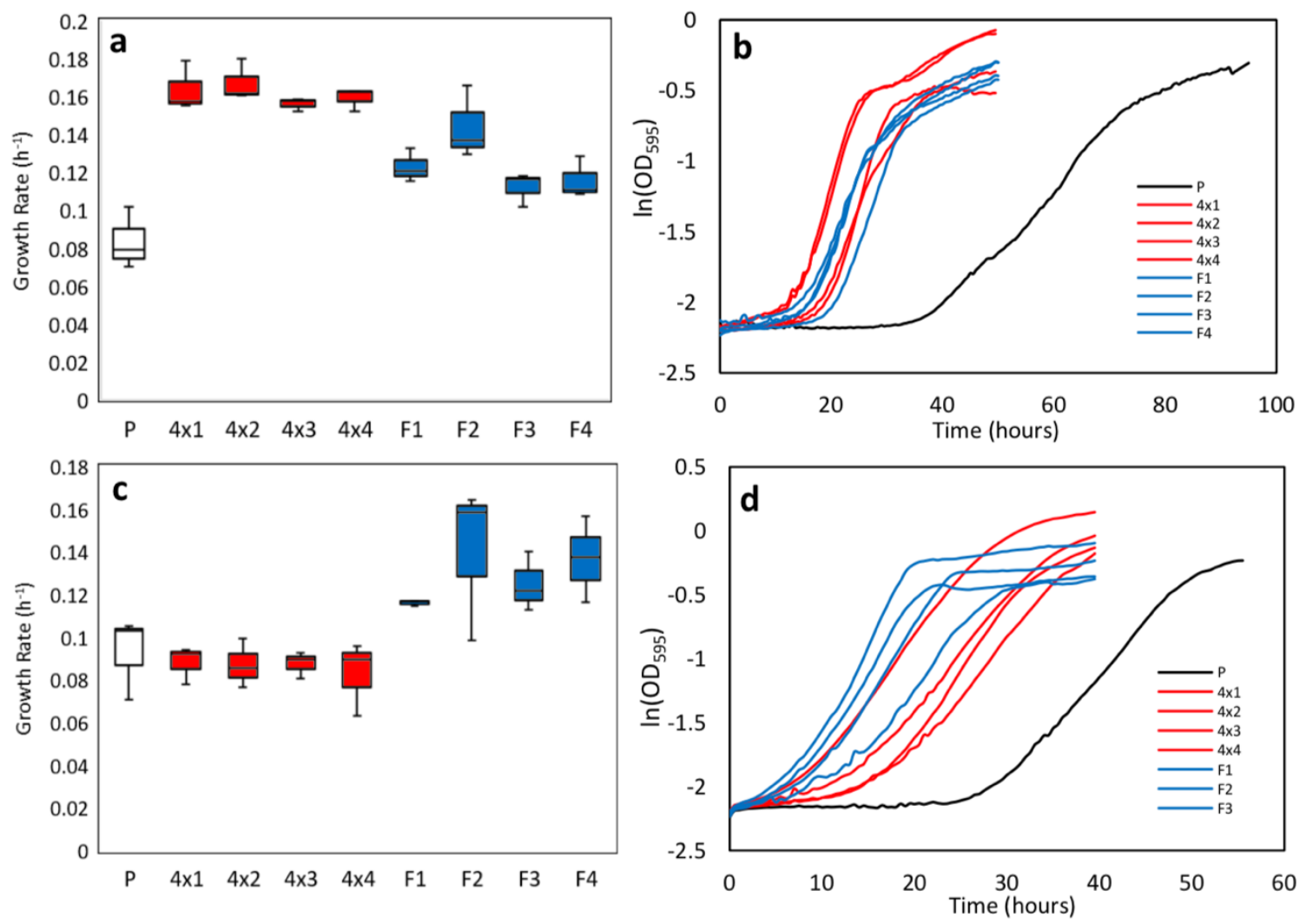

Fig. 5

Maximum growth rates (a) and plotted growth rates (b) of eight evolved NYC2580 strains and the progenitor in NLB + inhibitor cocktail. Inhibitor cocktail consisted of: acetic acid and furfural $1 \mathrm{~g} / \mathrm{L}$, formic acid and HMF $0.5 \mathrm{~g} / \mathrm{L}$. Maximum growth rates (c) and plotted growth rates (d) of eight mutant NYC2580 strains and the progenitor cultured in NLB $+0.85 \mathrm{~g} / \mathrm{L}$ formic acid. Each strain was assayed in triplicate with growth performed within a 96 well plate. Max growth rates were calculated using $\mathrm{R}$ Studio, curves represent the $\ln (O D)$ of each growth curve at 30 minute intervals

\section{Lipid production of evolved strains.}

Given that evolutionary trade-offs can be a significant draw back to ALE strain improvement, it is possible that the selection of inhibitor tolerant phenotypes could result in the loss of the oleaginous phenotype. To test for any effect on the lipid production, seven day cultures under inhibiting and noninhibiting conditions were performed for the evolved strains (Figure 6). Lipid production of the progenitor strain grown in NLB without inhibitors was slightly higher (22.1\%) than the four lineages evolved in formic acid (12.3-17.4\%). Lipid accumulation by the four-inhibitor cocktail evolved strains increased against the progenitor to yield between $32.5-41 \%$ lipid by dry weight. Furthermore, despite the comparable biomass of the formic acid evolved strains and the progenitor, all inhibitor cocktail evolved strains achieved a higher biomass, with the best performing strain reaching an average of 14.4 $\mathrm{g} / \mathrm{L}$. This result was also observed when all strains were cultured within NLB + inhibitor cocktail, with 
bioRxiv preprint doi: https://doi.org/10.1101/2020.02.17.952291; this version posted February $17,2020$. The copyright holder for this preprint (which was not certified by peer review) is the author/funder, who has granted bioRxiv a license to display the preprint in perpetuity. It is made available under aCC-BY-NC-ND 4.0 International license.

270

271

272

273

274

275

276

277

278

279

280

281

282

283

284

285

286

287

288

289

290

291

the inhibitor cocktail evolved strains outperforming the others, including one mutant reaching an average biomass of $8.5 \mathrm{~g} / \mathrm{L}$ with a lipid accumulation of $34.7 \%$. The progenitor in this media had reduced biomass $(4.3 \mathrm{~g} / \mathrm{L})$, but still achieved $20 \%$ lipid accumulation. In these conditions, however, the formic acid evolved strains had increased lipid production compared to when grown in control media (averaging 23\% versus 15\%), despite a lower biomass. Though formic acid and inhibitor cocktail evolved strains performed comparably within the growth assays, these results present strong evidence of unique adaptations occurring from each ALE strategy.
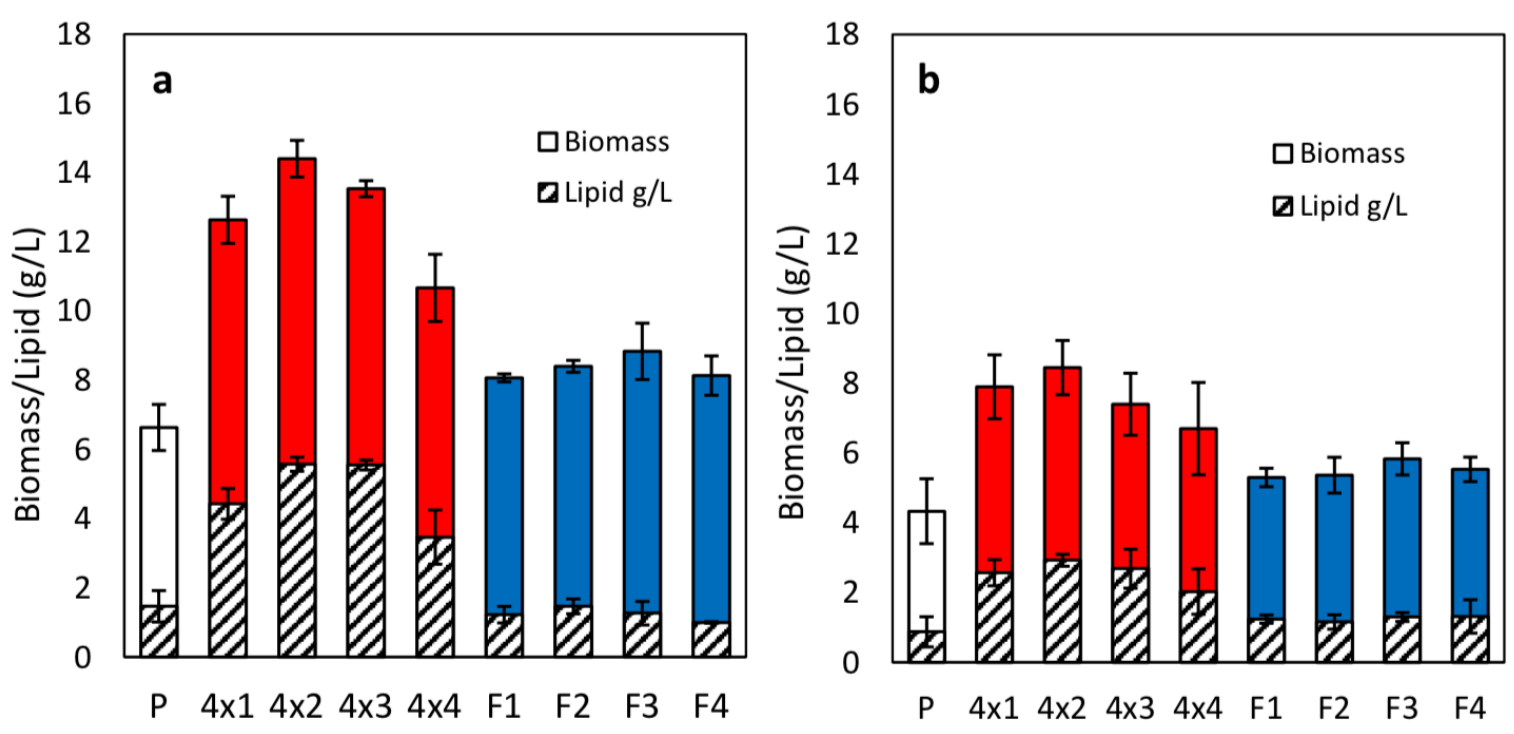

Fig. 6

Lipid quantification and biomass of evolved strains versus the progenitor grown in NLB (a) and NLB + Inhibitor cocktail (b). Inhibitor cocktail contained acetic acid and furfural $0.7 \mathrm{~g} / \mathrm{L}$, formic acid and HMF $0.35 \mathrm{~g} / \mathrm{L}$. Triplicate samples were cultured for each strain. Error bars values represent the standard deviation of the mean

Using the same samples assayed for lipid production, FAME profiling of all strains revealed further phenotypic differences. The FAME profile for the progenitor and inhibitor cocktail evolved strains varied very little when grown in the presence or absence of an inhibitor mix, though the latter had slightly increased ratios of $\mathrm{C} 16: 1$ and $\mathrm{C} 16: 0$ and lower amounts of $\mathrm{C} 18: 1$ when compared to the progenitor (Table 1). The formic acid evolved strains FAME profile varied considerably to the parental however, particularly when grown without inhibitors. Ratios of C16:0 decreased to an average of $10.6 \%$ across the four strains compared to $22.5 \%$ for the progenitor, and C18:1 and C18:2 levels increased to $78.5 \%$ and $8 \%$ on average compared to $71.3 \%$ and $2.5 \%$ respectively. 
FAME profiles of the progenitor and evolved strains.

\begin{tabular}{|c|cccccc|}
\hline NLB & C16:1 & C16:0 & C17:1 & C18:2 & C18:1 & C18:0 \\
\hline Progenitor & 3.1 & 22.5 & - & 2.5 & 71.3 & 0.5 \\
\hline $4 \times 1$ & 4.4 & 25.6 & - & 1.2 & 67.3 & 1.5 \\
$4 \times 2$ & 8.5 & 25.9 & 0.3 & 1.6 & 61.4 & 2.3 \\
$4 \times 3$ & 7.9 & 25.3 & 0.3 & 2.5 & 62.0 & 2.0 \\
$4 \times 4$ & 3.4 & 24.2 & - & 1.4 & 68.9 & 2.1 \\
\hline F1 & 1.2 & 11.1 & - & 5.8 & 81.2 & 0.7 \\
F2 & 2.4 & 12.3 & - & 7.2 & 77.2 & 0.8 \\
F3 & 1.2 & 8.1 & - & 11.8 & 77.6 & 1.3 \\
F4 & 3.2 & 11.0 & - & 7.2 & 78.2 & 0.5 \\
\hline
\end{tabular}

\begin{tabular}{|c|cccccc|}
\hline NLB + Inhibitors & C16:1 & C16:0 & C17:1 & C18:2 & C18:1 & C18:0 \\
\hline Progenitor & 1.9 & 22.0 & - & 1.8 & 73.2 & 1.1 \\
\hline $4 \times 1$ & 5.3 & 27.5 & 0.2 & 3.5 & 62.4 & 1.1 \\
$4 \times 2$ & 4.6 & 27.0 & - & 2.7 & 63.9 & 1.8 \\
$4 \times 3$ & 4.9 & 25.7 & - & 2.1 & 65.4 & 1.8 \\
$4 \times 4$ & 3.0 & 26.1 & - & 3.4 & 66.4 & 1.1 \\
\hline F1 & 1.9 & 17.6 & - & 1.7 & 74.9 & 3.9 \\
F2 & 2.6 & 17.1 & 0.5 & 1.7 & 75.5 & 2.7 \\
F3 & 3.0 & 16.1 & 0.7 & 1.0 & 76.7 & 2.5 \\
F4 & 3.3 & 16.1 & 0.8 & 1.1 & 76.4 & 2.3 \\
\hline
\end{tabular}

294

NLB + inhibitor cocktail (acetic acid and furfural $0.7 \mathrm{~g} / \mathrm{L}$, formic acid and HMF $0.35 \mathrm{~g} / \mathrm{L}$ ). Numbers represent fatty acid composition as \% of total fatty acids

\section{Influence of carbon-to-nitrogen ratio on lipid production in progenitor and evolved strains.}

Two strains; $4 \times 3$ and F3, were taken forward with the progenitor for further investigation into carbonto-nitrogen $(\mathrm{C}: \mathrm{N})$ response and how this has been affected by each directed evolution strategy. Lipid production was assayed within a full factorial ( 2 levels) experimental design, with carbon and nitrogen as the input factors. The $2 \mathrm{D}$ surface plot models the lipid production by weight percent for each strain in response to varying C:N within the experimental space. The response range (Lipid \%) for each plot ranges from the maximum and minimum lipid production values obtained for that individual strain, within the $\mathrm{C}: \mathrm{N}$ ratios assayed (Figure X). All strains exhibit a similar trend in response to varying $\mathrm{C}: \mathrm{N}$ ratios, accumulating the minimum amount of oil when nitrogen is high and carbon is low and maximum amounts when carbon is high and nitrogen low. The response plot for $4 \times 3$ immediately differs from $\mathrm{F} 3$ and the progenitor in continuing to accumulate a high amount of oil when carbon concentrations drop from their highest point, as shown by the larger red area. Another striking difference between all strains is the lipid production when both carbon and nitrogen are low (bottom 
bioRxiv preprint doi: https://doi.org/10.1101/2020.02.17.952291; this version posted February $17,2020$. The copyright holder for this preprint (which was not certified by peer review) is the author/funder, who has granted bioRxiv a license to display the preprint in perpetuity. It is made available under aCC-BY-NC-ND 4.0 International license.

left; $10 \mathrm{~g} / \mathrm{L}$ glucose, $0.3 \mathrm{~g} / \mathrm{L}$ ammonium sulphate). Here, $\mathrm{F} 3$ accumulates a low amount of oil relative to its maximum, $4 \times 3$ accumulates oil closer to its maximum and the progenitor comes in between the two. Finally, further differences are seen between the strains when comparing lipid production in top right and bottom left corners of the experimental space (high carbon, high nitrogen and low carbon, low nitrogen respectively). Here, the progenitor correlates with F3, producing a similar level of lipid at both conditions, whilst production is high for $4 \times 3$ when carbon and nitrogen concentrations are low, and decreased when concentrations are higher.

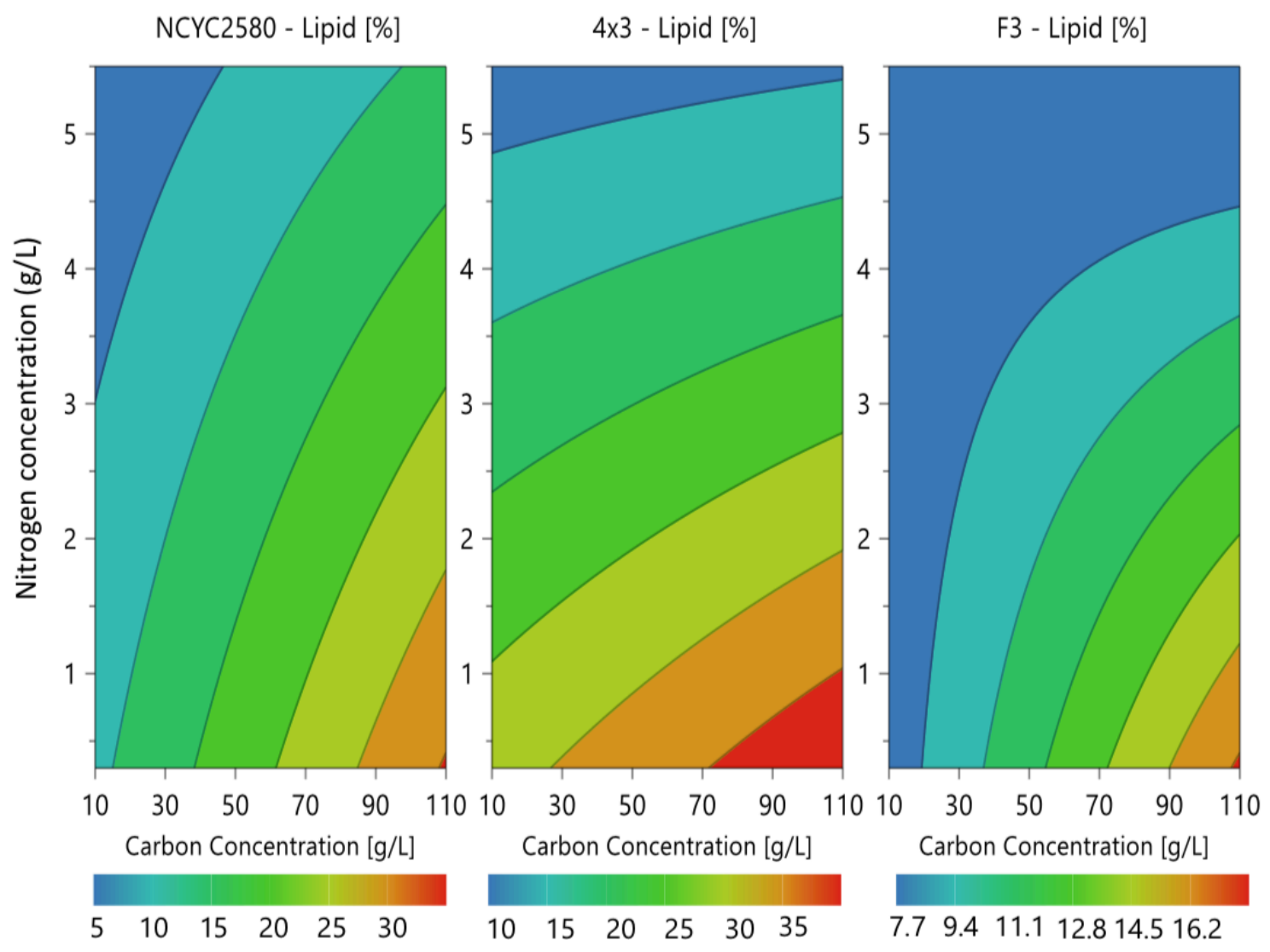

\section{Fig. 7}

2D surface plot from a full factorial ( 2 levels) experimental design with the progenitor NCYC2580 and evolved strains F3 and $4 \times 3$ assayed for lipid production in response to changing concentrations of carbon and nitrogen within YNB media for seven days. Each plot represents the maximum and minimum lipid production in weight percent for the individual strain, with the range split into seven equal contours.

\section{Culturing in $2 \mathrm{~L}$ bioreactor under non-sterile conditions}

To further assess the biotechnological potential of the evolved strains, the ' $4 \times 3$ ' strain evolved in the cocktail of inhibitors was taken forward for culturing in a $2 \mathrm{~L}$ controlled bioreactor under inhibiting and 
non-inhibiting conditions. Here, both cultures were performed in a non-sterile manner to best represent an industrial process. Final biomass within both bioreactor conditions exceeded that observed on the smaller scale, with control media conditions achieving $16 \mathrm{~g} / \mathrm{L}$ compared to $13.5 \mathrm{~g} / \mathrm{L}$ within tubes, and $14.5 \mathrm{~g} / \mathrm{L}$ versus $7.4 \mathrm{~g} / \mathrm{L}$ with the inhibitor containing media. The biomass increase is likely due to the high oxygenation supplied within the bioreactor ( $80 \% \mathrm{DO})$ and better gas transfer. This increase in biomass also suggests that increased oxygenation aids inhibitor detoxification leading to overall higher growth. Though final biomass at day seven within the control bioreactor was higher than within culture tubes, here, peak biomass of $17.5 \mathrm{~g} / \mathrm{L}$ was achieved after just 48 hours, giving a biomass productivity of $0.356 \mathrm{~g} \mathrm{~L}^{-1} \mathrm{~h}^{-1}$. A similar trend was also observed for lipid accumulation, where final values of $38.1 \%$ and $33.3 \%$ for control and inhibitor conditions respectively showed only a slight increase against 48 hour values of $35.1 \%$ and $29 \%$ in the tubes. This equates to a lipid productivity of $0.128 \mathrm{~g} \mathrm{~L}^{-1} \mathrm{~h}^{-1}$ and $0.088 \mathrm{~g} \mathrm{~L}^{-1} \mathrm{~h}^{-1}$ respectively.

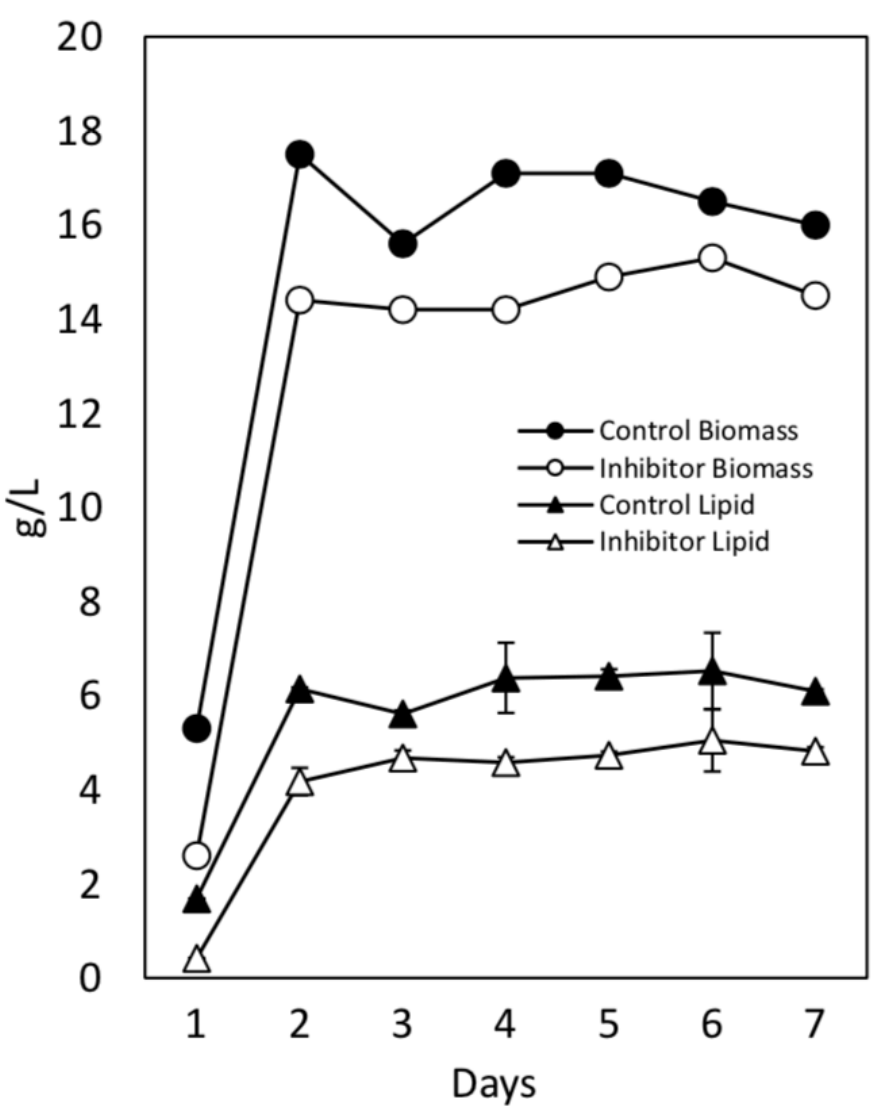

Fig. 8

Lipid quantification and biomass of strain ' $4 \times 3$ ' when grown in NLB (black circles/triangles) versus $\mathrm{NLB}+$ inhibitor cocktail (clear circles/triangles) under non-sterile bioreactor conditions. Inhibitor cocktail consisted of acetic acid and furfural $0.7 \mathrm{~g} / \mathrm{L}$, formic acid and HMF $0.35 \mathrm{~g} / \mathrm{L}$. Bioreactor was maintained at $20^{\circ} \mathrm{C}, \mathrm{pH} 4$ and $80 \%$ dissolved oxygen. Error bars for lipid production represent the 
Similarly to the smaller scale, the lipid profile did not change substantially when grown with or without inhibitors, however the level of saturated esters increased substantially compared with the tube cultures. For example, in the bioreactors the amount of $\mathrm{C} 16: 0$ and $\mathrm{C} 18: 1$ after 24 hours was $46 \%$ and $50 \%$ respectively against $25 \%$ and $62 \%$ when grown in the tubes (Table 2 ). This difference is presumably due to the increased oxygenation within the bioreactor.

Table 2. FAME profiles of the progenitor and evolved strains within bioreactors.

\begin{tabular}{|c|ccccc|}
\hline NLB & C16:1 & C16:0 & C18:2 & C18:1 & C18:0 \\
\hline Day 1 & 1.2 & 46.0 & 0.0 & 50.8 & 1.9 \\
Day 2 & 3.2 & 38.8 & 1.3 & 55.8 & 0.9 \\
Day 3 & 4.8 & 35.2 & 2.1 & 57.4 & 0.6 \\
Day 4 & 3.9 & 37.3 & 0.0 & 57.6 & 1.2 \\
Day 5 & 3.8 & 31.5 & 1.8 & 62.1 & 0.8 \\
Day 6 & 3.8 & 30.3 & 1.9 & 62.9 & 1.1 \\
Day 7 & 3.7 & 30.3 & 1.3 & 63.8 & 0.9 \\
\hline
\end{tabular}

\begin{tabular}{|c|ccccc|}
\hline NLB + Inhibitors & C16:1 & C16:0 & C18:2 & C18:1 & C18:0 \\
\hline Day 1 & 0.0 & 54.9 & 0.0 & 45.1 & 0.0 \\
Day 2 & 3.8 & 39.5 & 1.9 & 54.2 & 0.6 \\
Day 3 & 4.0 & 34.5 & 1.7 & 59.0 & 0.8 \\
Day 4 & 5.4 & 31.4 & 2.4 & 59.6 & 1.2 \\
Day 5 & 5.8 & 30.0 & 2.4 & 60.6 & 1.1 \\
Day 6 & 5.2 & 30.7 & 1.9 & 60.8 & 1.3 \\
Day 7 & 3.8 & 30.6 & 1.6 & 63.3 & 0.8 \\
\hline
\end{tabular}

$\mathrm{NLB}+$ inhibitor cocktail (acetic acid and furfural $0.7 \mathrm{~g} / \mathrm{L}$, formic acid and HMF $0.35 \mathrm{~g} / \mathrm{L}$ ). Numbers

\section{Discussion}

\section{Adaptive Evolution of NCYC2580.}

The 48 hour batch transfer experimental design used within this study aimed to maintain cells in exponential phase as much as practically possible. In this way, stationary phase adaptation was to be minimised, and cells were exposed to a 'fresh' dose of inhibitors when stress tolerance is generally at its lowest (Plesset et al. 1987). The short batch time was also designed to specifically select for two industrially advantageous phenotypes; high growth rate and low lag time under inhibiting conditions, with slower growing populations eventually dropping out due to successive low frequencies at transfer. This approach however, is not favourable for selecting survival adaptations once the stationary phase was reached. 
Differences between both evolution strategies were stark; inhibitor concentrations in the formic acid strategy gradually increased to $1.2 \mathrm{~g} / \mathrm{L}$ from a starting point of $0.6 \mathrm{~g} / \mathrm{L}$ whilst the starting concentration within the inhibitor cocktail strategy was maintained for the duration and lineages were frequently lost due to batch death. This result is most likely due to the multitude of stresses exerted by the synergistic effect of inhibitors (Martín et al. 2007). Successful ALE experiments using multi-inhibitor containing lignocellulosic hydrolysate or synthetic media containing inhibitor cocktails using a sequential batch approach are limited within the literature. Rather, studies of this kind are performed within chemostats where the maintenance of biomass leads to quicker detoxification of inhibitors by reducing the dose per cell effect (Martín et al. 2007; Smith et al. 2014). A sequential batch strategy applied by Koppram et al. in agreement with results within Figure 3Error! Reference source not found., also encountered early complications, reporting a lag time increase of 35 to 50 hours within the initial batches (Koppram et al. 2012). However, as batch transfers were performed upon depletion of glucose within the media opposed to at a set time point as presented here, it is likely that this strategy is not targeted at selecting for mutants with reduced lag time or increased growth rate unlike the rationale presented here.

\section{Growth Analysis of Evolved Strains.}



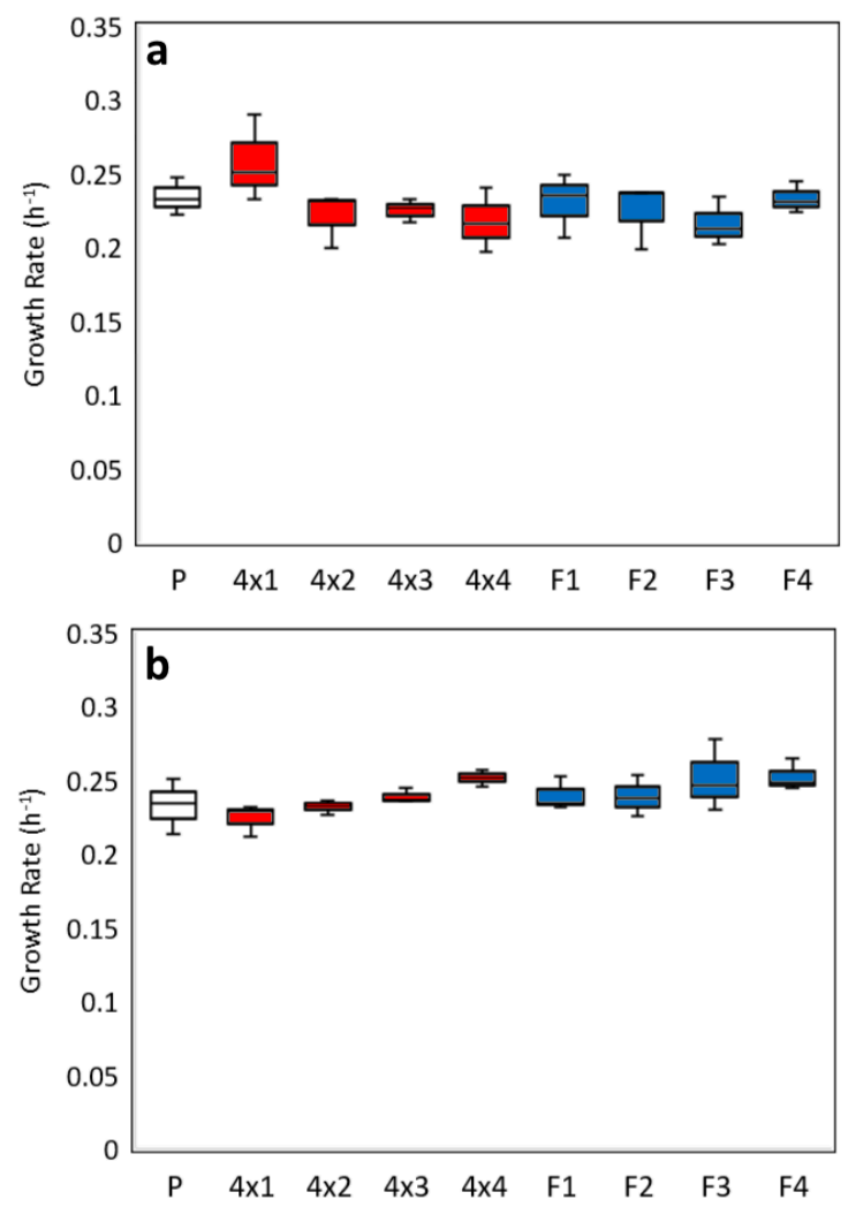

Fig. ), it is improbable that the ALE strategies have caused advantageous mutations or copy number amplifications that increase glycolytic efficiencies leading to decreased lag time and increased growth rate. Similarly, it is unlikely that the evolved strains are faster growing due to increased glucose transportation efficiencies as has been observed within S. cerevisiae strains evolved to glucose-limited media (Brown et al. 1998; Dunham et al. 2002). Rather, given that growth rate improvements were only observed under inhibiting conditions, then any adaptive improvements are likely to be specific to inhibitor tolerance. Mutation or amplification candidates aiding acid tolerance include multi-drug transporters or proteins within the ABC transporter family such as Pdr12 or Azr1, the latter of which has been shown to contribute to acetic acid tolerance (Piper et al. 1998; Tenreiro et al. 2000). Increased tolerance and growth within furfural and HMF containing medias on the other hand could implicate the efficiency or abundance of furfural/HMF reducing enzymes such as Adh1, Adh6 and Sfa1 (Petersson et al. 2006). Though there are limited examples of genomic sequencing applied to strains evolved to fermentation inhibitors, Sako et al. found both a loss of heterozygosity within one chromosomal arm and duplication within another within an engineered S. cerevisiae strain evolved to inhibitor containing media, with both events involving members of the PDR ABC transporter gene family (Sato et al. 2014). The loss of heterozygosity event occurred within the chromosomal arm 
containing $P D R 1$, a gene regulating the expression of multidrug resistance transporters including those associated with tolerance to gallic acid, whereas the duplication event contained PDR18, a gene important for tolerance to herbicides and metal ions, PDR16, a regulator of lipid biosynthesis and PDR17, a regulator of membrane composition.

As two distinct evolution strategies were performed, it was anticipated that mutants will develop adaptations specific to their own evolution condition. In this respect, as the inhibitor cocktail contained both acid and aldehyde inhibitors, it was expected that these mutants would perform well when assayed in just formic acid supplemented media which was not observed. Conversely, mutants evolved to formic acid performed strongly when assayed with an inhibitor cocktail. This is particularly surprising given the presence of HMF is known to induce long lag times in S. cerevisiae (Sehnem et al. 2013). These results favour the emergence of non-specific mutations to confer increased tolerance, such as the upregulation of genes such as WHI2 encoding for a protein required to activate the general stress response and shown to increase tolerance to acetic acid and furfural both individually and in combination when overexpressed (Chen et al. 2016).

\section{Lipid Production of Evolved Strains.}

Though presence of inhibitors, whether as a single supplemented inhibitor or an inhibitor cocktail, is reported elsewhere to reduce biomass in oleaginous yeasts as shown here, their effect on lipid production varies (Chen et al. 2009; Hu et al. 2009; Yu et al. 2011). Within Rhodosporidium toruloides for example (Hu et al. 2009), no effect on lipid production was observed when cultured in the presence of six inhibitors, whereas the lipid accumulation of $C$. curvatus has been shown to reduce by $62 \%$ when in the presence of $1 \mathrm{~g} / \mathrm{L}$ furfural (Yu et al. 2011). In this study, the lipid production of the three groups of strains were affected by inhibitors in different ways: the progenitor was unaffected, the formic acid evolved strains increased lipid production and the inhibitor cocktail evolved strains had their production reduced. Despite this, certainly the most striking result was the overall lipid production of inhibitor cocktail evolved strains increasing compared with the progenitor. Furthermore, as the formic acid evolved strains did not share this additional phenotype, it is likely that adaptation to the aldehyde inhibitors is responsible. An indication as to what adaptations may have occurred when evolving to an cocktail containing aldehyde inhibitors are given by how these compounds are processed by the cells. Within S. cerevisiae, furfural and HMF are both converted into less toxic compounds by NADPHdependant enzymes (Petersson et al. 2006; Heer et al. 2009) meaning evolution to media containing both may have selected for adaptations to increase cellular concentrations of NADPH for quicker inhibitor detoxification. Mutations or gene copy number amplifications of $P O S 5$, responsible for the conversion of NADH to NADPH within the mitochondria, or ALD6 which converts NADP ${ }^{+}$into NADPH 
within the cytosol are therefore potential targets (Miyagi et al. 2009). NADPH also plays a dual role within oleaginous organisms, functioning as a critical component in fatty acid synthesis. Despite it traditionally believed that NADPH used during fatty acid synthesis was derived solely from the activity of a cytosolic malic enzyme, recent studies have revealed that NADPH produced elsewhere within the cell can also be used to this end. These include the activities of glucose-6-phosphate dehydrogenase (G6PD) or 6-phosphogluconate dehydrogenase (PGD) in the pentose phosphate pathway, meaning that several alternative NADPH sources could be involved in the increased lipogenesis observed by these strains (Zhang et al. 2007; Chen et al. 2015). Given this, it is possible therefore that if a mutation has caused cellular levels of NADPH to be greater than that of the progenitor, particularly in conditions where inhibitors are not present, that fatty acid synthesis is providing a 'sink' for the excess NADPH. Furthermore, if these mutations were combined with a gene duplication to the lipid biosynthesis regulator PDR16 as was observed within a S. cerevisiae strain evolved to fermentation inhibitors (Sato et al. 2014), then their synergistic effect may be responsible for this new phenotype.

An alternate explanation for increased lipid production within the multi inhibitor evolved strains could be due to an altered C:N response. Applying an experimental design to assay $\mathrm{C}: \mathrm{N}$ response of two evolved strains, $4 \times 3$ and F3, revealed distinct differences from its progenitor stage. In particular, high lipid accumulation by $4 \times 3$ was shown to occur over a broader range of $\mathrm{C}: \mathrm{N}$ ratios assayed compared to the progenitor, with the reverse true for strain F3. As both strategies took place within nitrogen limited media, evolution to display a nitrogen limited, and therefore lipid rich, phenotype at lower ratios of carbon to nitrogen does not seem to have occurred due to the dissimilar C:N response by these two evolved strains. Again therefore it suggests that adaptation to the inhibitors themselves, rather than adaptation to nitrogen limited media, is responsible for the altered $\mathrm{C}: \mathrm{N}$ response of $4 \times 3$. Indeed, it is reported that genes indicating nitrogen starvation were up regulated in S. cerevisiae in response to HMF and furfural supplemented media, with it further stated that NADPH flux usually directed towards ammonium assimilation under control conditions was instead redirected to detoxification of HMF and furfural (Ask et al. 2013).

Although overall lipid production was influenced by the presence of inhibitors (Figure 6), the FAME profile for all strains remained relatively unchanged (Table 1), agreeing with work by Hu et al. and Yu et al. (Hu et al. 2009; Yu et al. 2011). What did affect the FAME profile however was culture, age as shown within the non-sterile bioreactor cultures (Table 2). Under both inhibiting and non-inhibiting conditions, initially high levels of C16:0 within the first 48 hours of growth reduced and C18:1 and C16:1 levels increased as the cultures progressed. Here, it is likely that C16:0 fatty acids are either 
Amaro and Nicaud 2016), or further elongated (either before or after desaturation to C16:1) to C18:1. Maintaining a more saturated lipid, suitable as a palm oil substitute, would therefore require the cessation of culturing after 48 hours, or targeting enzymes such as OLE1 for deletion. Whereas if a more monounsaturated product was required, for biodiesel production, then the yeast would need a longer time in the bioreactor.

474 The strain presented in this study appears unique amongst oleaginous organisms in producing a 475 microbial oil high in C16:0, with Cryptococcus curvatu (28\%), R. glutinis (18\%) and Y. lipolytica (11\%) 476 having a notably lower proportion (Ageitos et al. 2011). The narrow FAME profile of this organism, consisting almost exclusively of C16:0 and C18:1 fatty acids, also appears contrary to other oleaginous yeast. The FAME profile of $Y$. lipolytica and Trichosporon pullulans for example is reported to consist $51 \%$ and $24 \%$ of C18:2 respectively, whilst $C$. curvatus and $R$. graminis produce $15 \%$ and $12 \%$ C18:0; FAME's M. pulcherrima appears to produce only in trace amounts (Beopoulos et al. 2009; Ageitos et al. 2011).

This study emphasises the effectiveness of adaptive evolution to generate improved phenotypes in instances where genetic engineering is either not available, or, as with the aims of this study, difficult to approach with rational design due to the vast network of molecular mechanisms. By employing different strategies, though both successful with respect to increasing inhibitor tolerance, adaptive evolution can provide strain improvements beyond the initial aim.

\section{Acknowledgments}

This research has been funded by the Industrial Biotechnology Catalyst (Innovate UK, BBSRC, EPSRC) to support the translation, development and commercialisation of innovative Industrial Biotechnology processes (EP/N013522/1) and by the EPSRC through the Centre for Doctoral Training in Sustainable Chemical Technologies (EP/L016354/1).

\section{Author Contributions}

ALE was planned by RHH and performed by YS. RHH planned and conducted all further work, and wrote the manuscript. CJC and DAH advised the study, and revised the manuscript. All authors read and approved the final manuscript. 
References:

500

501

502

503

504

505

506

507

508

509

510

511

512

Ageitos JM, Vallejo JA, Veiga-Crespo P, Villa TG (2011) Oily yeasts as oleaginous cell factories. Appl. Microbiol. Biotechnol. 90:1219-1227

Ask M, Bettiga M, Mapelli V, Olsson L (2013) The influence of HMF and furfural on redox-balance and energy-state of xylose-utilizing Saccharomyces cerevisiae. Biotechnol Biofuels 6: . doi: $10.1186 / 1754-6834-6-22$

Beopoulos A, Cescut J, Haddouche R, Uribelarrea JL, Molina-Jouve C, Nicaud JM (2009) Yarrowia lipolytica as a model for bio-oil production. Prog. Lipid Res. 48:375-387

Bligh EG, Dyer WJ (1959) A rapid method of total lipid extraction and purification. Can J Biochem Physiol 37:911-917 . doi: 10.1139/o59-099

Brown CJ, Todd KM, Rosenzweig RF (1998) Multiple duplications of yeast hexose transport genes in response to selection in a glucose-limited environment. Mol Biol Evol 15:931-942 . doi: 10.1093/oxfordjournals.molbev.a026009

Chen H, Hao G, Wang L, Wang H, Gu Z, Liu L, Zhang H, Chen W, Chen YQ (2015) Identification of a critical determinant that enables efficient fatty acid synthesis in oleaginous fungi. Sci Rep 5:11247 . doi: 10.1038/srep11247

Chen X, Li Z, Zhang X, Hu F, Ryu DDY, Bao J (2009) Screening of oleaginous yeast strains tolerant to lignocellulose degradation compounds. In: Applied Biochemistry and Biotechnology. pp 591604

Chen Y, Sheng J, Jiang T, Stevens J, Feng X, Wei N (2016) Transcriptional profiling reveals molecular basis and novel genetic targets for improved resistance to multiple fermentation inhibitors in Saccharomyces cerevisiae. Biotechnol Biofuels 9:1-18 . doi: 10.1186/s13068-015-0418-5

Dragosits M, Mattanovich D (2013) Adaptive laboratory evolution--principles and applications for biotechnology. Microb Cell Fact 12:64

Dunham MJ, Badrane H, Ferea T, Adams J, Brown PO, Rosenzweig F, Botstein D (2002) Characteristic genome rearrangements in experimental evolution of Saccharomyces cerevisiae. Proc Natl Acad Sci 99:16144-16149 . doi: 10.1073/pnas.242624799

Fan J, Santamauro F, Budarin V, Whiffin F, Abeln F, Chantasuban T, Gore-Lloyd D, Henk D, Scott RJ, Clark J, Chuck CJ (2018) The additive free microwave hydrolysis of lignocellulosic biomass for 
fermentation to high value products. J Clean Prod 198:776-784 . doi: 10.1016/j.jclepro.2018.07.088

Field SJ, Ryden P, Wilson D, James S a, Roberts IN, Richardson DJ, Waldron KW, Clarke T a (2015) Identification of furfural resistant strains of Saccharomyces cerevisiae and Saccharomyces paradoxus from a collection of environmental and industrial isolates. Biotechnol Biofuels 8:33 . doi: 10.1186/s13068-015-0217-z

Heer D, Heine D, Sauer U (2009) Resistance of Saccharomyces cerevisiae to high concentrations of furfural is based on NADPH-dependent reduction by at least two oxireductases. Appl Environ Microbiol 75:7631-7638 . doi: 10.1128/AEM.01649-09

Hu C, Zhao X, Zhao J, Wu S, Zhao ZK (2009) Effects of biomass hydrolysis by-products on oleaginous yeast Rhodosporidium toruloides. Bioresour Technol 100:4843-4847 . doi:

$$
\text { 10.1016/j.biortech.2009.04.041 }
$$

Klinke HB, Thomsen AB, Ahring BK (2004) Inhibition of ethanol-producing yeast and bacteria by degradation products produced during pre-treatment of biomass. Appl. Microbiol. Biotechnol. $66: 10-26$

Koppram R, Albers E, Olsson L (2012) Evolutionary engineering strategies to enhance tolerance of xylose utilizing recombinant yeast to inhibitors derived from spruce biomass. Biotechnol Biofuels 5:32 . doi: 10.1186/1754-6834-5-32

Kurosawa K, Laser J, Sinskey AJ (2015) Tolerance and adaptive evolution of triacylglycerol-producing Rhodococcus opacus to lignocellulose-derived inhibitors. Biotechnol Biofuels 8:76 . doi: $10.1186 / \mathrm{s} 13068-015-0258-3$

Ledesma-Amaro R, Nicaud JM (2016) Yarrowia lipolytica as a biotechnological chassis to produce usual and unusual fatty acids. Prog. Lipid Res. 61:40-50

Lin FM, Qiao B, Yuan YJ (2009) Comparative proteomic analysis of tolerance and adaptation of ethanologenic Saccharomyces cerevisiae to furfural, a lignocellulosic inhibitory compound. Appl Environ Microbiol 75:3765-3776 . doi: 10.1128/AEM.02594-08

Long Z, Santomauro F, Fan J, Macquarrie D, Clark J, Chuck CJ, Budarin V (2017) Fast microwaveassisted acidolysis: a new biorefinery approach for the zero-waste utilisation of lignocellulosic biomass to produce high quality lignin and fermentable saccharides. Faraday Discuss. doi: 10.1039/c7fd00102a 
Martín C, Marcet M, Almazán O, Jönsson ப (2007) Adaptation of a recombinant xylose-utilizing Saccharomyces cerevisiae strain to a sugarcane bagasse hydrolysate with high content of fermentation inhibitors. Bioresour Technol 98:1767-1773 . doi: 10.1016/j.biortech.2006.07.021

Miyagi H, Kawai S, Murata K (2009) Two sources of mitochondrial NADPH in the yeast Saccharomyces cerevisiae. J Biol Chem 284:7553-7560 . doi: 10.1074/jbc.M804100200

Petersson A, Almeida JRM, Modig T, Karhumaa K, Hahn-Hägerdal B, Gorwa-Grauslund MF, Lidén G (2006) A 5-hydroxymethyl furfural reducing enzyme encoded by the Saccharomyces cerevisiae ADH6 gene conveys HMF tolerance. Yeast 23:455-464 . doi: 10.1002/yea.1370

Piper $\mathrm{P}$, Mahé $\mathrm{Y}$, Thompson S, Pandjaitan R, Holyoak $\mathrm{C}$, Egner R, Mühlbauer M, Coote $\mathrm{P}$, Kuchler $\mathrm{K}$ (1998) The Pdr12 ABC transporter is required for the development of weak organic acid resistance in yeast. EMBO J 17:4257-4265 . doi: 10.1093/emboj/17.15.4257

Plesset J, Ludwig JR, Cox BS, McLaughlin CS (1987) Effect of cell cycle position on thermotolerance in Saccharomyces cerevisiae. J Bacteriol 169:779-784

Radecka D, Mukherjee V, Mateo RQ, Stojiljkovic M, Foulquié-Moreno MR, Thevelein JM (2015) Looking beyond Saccharomyces: The potential of non-conventional yeast species for desirable traits in bioethanol fermentation. FEMS Yeast Res. 15

RStudio (2015) RStudio: Integrated Development for R

Santamauro F, Whiffin FM, Scott RJ, Chuck CJ (2014) Low-cost lipid production by an oleaginous yeast cultured in non-sterile conditions using model waste resources. Biotechnol Biofuels 7:34 . doi: $10.1186 / 1754-6834-7-34$

Sato TK, Liu T, Parreiras LS, Williams DL, Wohlbach DJ, Bice BD, Ong IM, Breuer RJ, Qin L, Busalacchi D, Deshpande S, Daum C, Gasch AP, Hodge DB (2014) Harnessing genetic diversity in saccharomyces cerevisiae for fermentation of xylose in hydrolysates of alkaline hydrogen peroxide-pretreated biomass. Appl Environ Microbiol 80:540-554 . doi: 10.1128/AEM.0188513

Sehnem NT, da Silva Machado A, Leite FCB, de Barros Pita W, de Morais MA, Ayub MAZ (2013) 5Hydroxymethylfurfural induces ADH7 and ARI1 expression in tolerant industrial Saccharomyces cerevisiae strain P6H9 during bioethanol production. Bioresour Technol 133:190-196 . doi: 10.1016/j.biortech.2013.01.063 
yeasts for biodiesel: Current and future trends in biology and production. Biotechnol. Adv.

Smith J, van Rensburg E, Görgens JF (2014) Simultaneously improving xylose fermentation and tolerance to lignocellulosic inhibitors through evolutionary engineering of recombinant Saccharomyces cerevisiae harbouring xylose isomerase. BMC Biotechnol 14:41 . doi: $10.1186 / 1472-6750-14-41$

Stanley D, Fraser S, Chambers PJ, Rogers P, Stanley GA (2010) Generation and characterisation of stable ethanol-tolerant mutants of Saccharomyces cerevisiae. J Ind Microbiol Biotechnol 37:139-149 . doi: 10.1007/s10295-009-0655-3

Taylor MP, Mulako I, Tuffin M, Cowan D (2012) Understanding physiological responses to pretreatment inhibitors in ethanologenic fermentations. Biotechnol. J. 7:1169-1181

Tenreiro S, Rosa PC, Viegas CA, Sá-Correia I (2000) Expression of the AZR1 gene (ORF YGR224w), encoding a plasma membrane transporter of the major facilitator superfamily, is required for adaptation to acetic acid and resistance to azoles in Saccharomyces cerevisiae. Yeast 16:14691481

Van Maris AJA, Winkler AA, Kuyper M, De Laat WTAM, Van Dijken JP, Pronk JT (2007) Development of efficient xylose fermentation in saccharomyces cerevisiae: Xylose isomerase as a key component. Adv Biochem Eng Biotechnol 108:179-204 . doi: 10.1007/10_2007_057

Whiffin F, Santomauro F, Chuck CJ (2016) Toward a microbial palm oil substitute: Oleaginous yeasts cultured on lignocellulose. Biofuels, Bioprod. Biorefining

Wisselink HW, Toirkens MJ, Berriel MDRF, Winkler AA, Van Dijken JP, Pronk JT, Van Maris AJA (2007) Engineering of Saccharomyces cerevisiae for efficient anaerobic alcoholic fermentation of Larabinose. Appl Environ Microbiol 73:4881-4891 . doi: 10.1128/AEM.00177-07

Wright J, Bellissimi E, de Hulster E, Wagner A, Pronk JT, Van Maris AJA (2011) Batch and continuous culture-based selection strategies for acetic acid tolerance in xylose-fermenting Saccharomyces cerevisiae. FEMS Yeast Res 11:299-306 . doi: 10.1111/j.1567-1364.2011.00719.x

Yu X, Zheng Y, Dorgan KM, Chen S (2011) Oil production by oleaginous yeasts using the hydrolysate from pretreatment of wheat straw with dilute sulfuric acid. Bioresour Technol 102:6134-6140 . doi: 10.1016/j.biortech.2011.02.081 
bioRxiv preprint doi: https://doi.org/10.1101/2020.02.17.952291; this version posted February $17,2020$. The copyright holder for this

preprint (which was not certified by peer review) is the author/funder, who has granted bioRxiv a license to display the preprint in perpetuity. It is made available under aCC-BY-NC-ND 4.0 International license. accumulation. Microbiology 153:2013-2025 . doi: 10.1099/mic.0.2006/002683-0 